\title{
Study of Polarized ep Collisions and Combined Electroweak and QCD Fits at HERA
}

\author{
Vladimir Chekelian (Shekelyan)* \\ on behalf of the HI and ZEUS Collaborations \\ MPI for Physics, Munich \\ E-mail: shekeln@mppmu.mpg.de
}

\begin{abstract}
The $e p$ inclusive deep inelastic cross sections are measured in the H1 and ZEUS experiments for the neutral and charged current processes in interactions with longitudinally polarized lepton beams. The polarization dependences of the charged current and polarization asymmetries of the neutral current $e^{+} p$ and $e^{-} p$ cross sections are presented. The lepton charge asymmetry of the unpolarised NC cross sections is used to extract the structure function $x F_{3}^{\gamma Z}$. Combined electroweak and QCD analyses of the polarized HERA data are performed to determine electroweak parameters taking their correlation with parton distributions into account. The couplings of the light $u$ and $d$ quarks to the $Z^{0}$-boson are determined and compared to the results from the LEP and the Tevatron.
\end{abstract}

35th International Conference of High Energy Physics - ICHEP2010,

July 22-28, 2010

Paris France

* Speaker. 


\section{Introduction}

At the ep collider HERA (1992-2007), electrons of $27.5 \mathrm{GeV}$ collided with protons of predominantly $920 \mathrm{GeV}$. This corresponds to an ep center of mass energy of $320 \mathrm{GeV}$. After the luminosity upgrade of the machine in the second phase of the HERA program (HERA-II, 2003-2007), the accelerator provided longitudinal polarization of the lepton beams (of about 40\%) defined as $P=\left(N_{R}-N_{L}\right) /\left(N_{R}+N_{L}\right)$, where $N_{R}\left(N_{L}\right)$ is the number of right- (left-)handed leptons in the beam. For that purpose pairs of spin rotators were implemented in the beam-line on either side of the interaction regions, which rotated transversely polarized leptons into longitudinally polarized states and back again. The transverse polarization of the lepton beam arised naturally through synchrotron radiation via the Sokolov-Ternov effect.

The longitudinal polarization of the lepton beam at HERA-II was exploited in the measurements of the $e^{+} p$ and $e^{-} p$ inclusive neutral (NC) and charged (CC) current deep inelastic scattering cross sections by H1 [1] and ZEUS [2]. The $e^{-} p$ and $e^{+} p$ data with negative (" $L$ ") and positive (" $R$ ") polarizations are also combined together to provide measurements of the unpolarized $e^{ \pm} p$ cross sections. In order to improve the statistical precision, the $\mathrm{H} 1$ cross section measurements are combined with previously published measurements from HERA-I representing the complete $\mathrm{H} 1$ data set. The unpolarized $e^{ \pm} p \mathrm{NC}$ and $\mathrm{CC}$ cross sections are shown in Fig. 1 (left). Both H1 and ZEUS performed combined electroweak (EW) and QCD analyses of the $\mathrm{NC}$ and $\mathrm{CC}$ data to determine electroweak parameters taking their correlation with parton distributions into account.

\section{CC cross sections with longitudinally polarized leptons}

The polarization dependence of the $\mathrm{CC} e^{ \pm} p \rightarrow v(\bar{v}) X$ cross sections is calculable within the Standard Model (SM) framework. The SM predicts that in the absence of right-handed charged currents, the $e^{+} p\left(e^{-} p\right)$ CC cross section is directly proportional to the fraction of right-handed positrons (left-handed electrons) in the beam. Thus, it can be expressed as

$$
\sigma_{C C}^{e^{ \pm} p}(P)=(1 \pm P) \sigma_{C C}^{e^{ \pm} p}(P=0) .
$$

The total CC cross sections $\sigma_{C C}^{e^{ \pm} p}$ measured by $\mathrm{H} 1$ and ZEUS for negative four-momentum transfers squared $Q^{2}>400 \mathrm{GeV}^{2}$ and inelasticity $y<0.9$ are shown in Fig. 1 (right). The measurements of the unpolarized total cross section based on the HERA-I data are also shown. The measurements exhibit the expected linear dependence as a function of the polarization and are consistent with the axiomatic absence of right-handed charged currents in the Standard Model.

\section{NC cross sections with longitudinally polarized leptons}

The cross section of the NC process, $e^{ \pm} p \rightarrow e^{ \pm} X$, can be written as

$$
\frac{\mathrm{d}^{2} \sigma_{\mathrm{NC}}^{\mathrm{e}^{ \pm} \mathrm{p}}}{\mathrm{dxd \textrm {Q } ^ { 2 }}}=\frac{2 \pi \alpha^{2}}{x Q^{4}} Y_{+} \tilde{\sigma}_{N C}^{ \pm}\left(x, Q^{2}\right) \equiv \frac{2 \pi \alpha^{2}}{x Q^{4}}\left(Y_{+} \tilde{F}_{2}^{ \pm} \mp Y_{-} x \tilde{F}_{3}^{ \pm}-y^{2} \tilde{F}_{L}^{ \pm}\right),
$$

where $\alpha$ is the fine structure constant and $x$ is the Bjorken scaling variable. The helicity dependences are contained in $Y_{ \pm}=1 \pm\left(1-y^{2}\right)$ and $\tilde{\sigma}_{N C}^{ \pm}$is the reduced cross section. The first two 

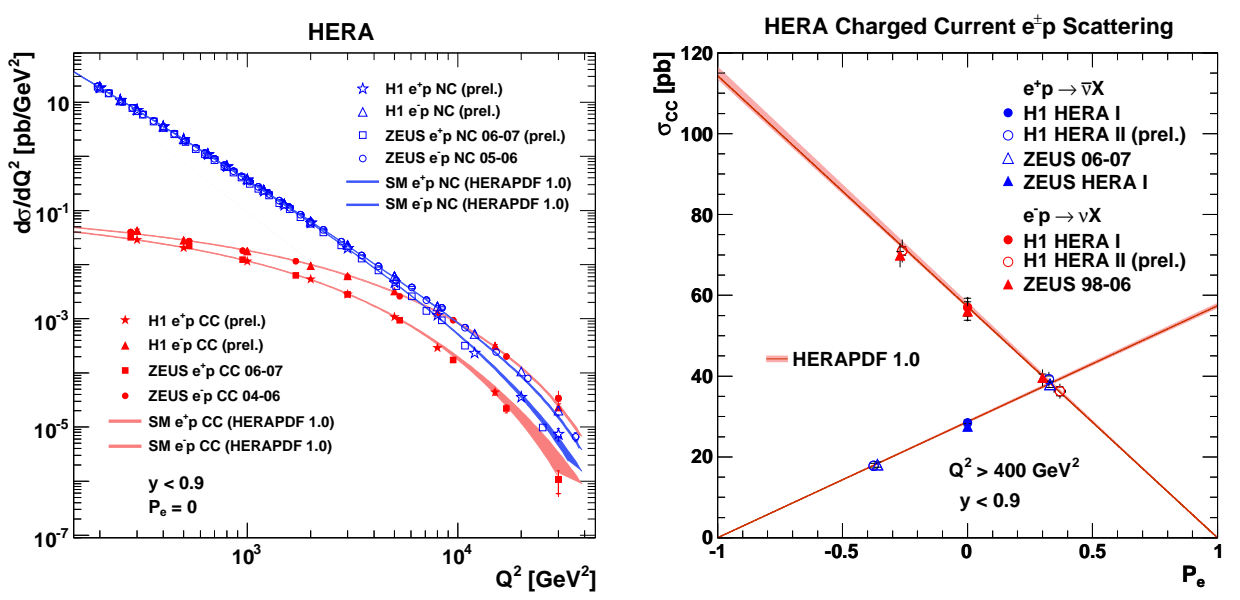

Figure 1: Left: The $e^{+} p$ and $e^{-} p$ NC and CC unpolarized cross sections. Right: The dependence of the $e^{+} p$ and $e^{-} p$ CC cross sections on the lepton beam polarization $P$. The data are compared to the Standard Model prediction based on the HERAPDF1.0 [3] parameterization.

generalized proton structure functions can be decomposed as

$$
\begin{array}{cc}
\tilde{F}_{2}^{ \pm} & =F_{2}+k\left(-v_{e} \mp P a_{e}\right) F_{2}^{\gamma Z}+k^{2}\left(v_{e}^{2}+a_{e}^{2} \pm 2 P v_{e} a_{e}\right) F_{2}^{Z}, \\
x \tilde{F}_{3}^{ \pm} & =k\left(-a_{e} \mp P v_{e}\right) x F_{3}^{\gamma Z}+k^{2}\left(2 v_{e} a_{e} \pm P\left(v_{e}^{2}+a_{e}^{2}\right)\right) x F_{3}^{Z},
\end{array}
$$

where $k$ is scheme dependent and takes the form $k^{-1}=\frac{2 \sqrt{2} \pi \alpha}{G_{F} M_{Z}^{2}} \frac{Q^{2}+M_{Z}^{2}}{Q^{2}}$ in the modified on-mass-shell scheme [4]. $M_{Z}$ is the $Z^{0}$ mass and $G_{F}$ is the Fermi constant. The quantities $v_{e}=-1 / 2+2 \sin ^{2} \theta_{W}$ and $a_{e}=-1 / 2$ are the vector and axial-vector couplings of the electron to the $Z$ boson with $\theta_{W}$ being the electroweak mixing angle (Weinberg angle). The third generalized structure function $\tilde{F}_{L}^{ \pm}$ is important only at high $y$ and is expected to be negligible at large $x$ and $Q^{2}$.

At leading order in QCD, $F_{2}, F_{2}^{\gamma Z}, F_{2}^{Z}$ are related to linear combinations of sums and differences of the quark and anti-quark momentum distributions $x q$ and $x \bar{q}$ of the proton:

$$
\begin{gathered}
\left(F_{2}, F_{2}^{\gamma Z}, F_{2}^{Z}\right)=x \sum\left(e_{q}^{2}, 2 e_{q} v_{q}, v_{q}^{2}+a_{q}^{2}\right)(q+\bar{q}), \\
\left(x F_{3}^{\gamma Z}, x F_{3}^{Z}\right)=2 x \sum\left(e_{q} a_{q}, v_{q} a_{q}\right)(q-\bar{q}),
\end{gathered}
$$

where $v_{q}$ and $a_{q}$ are the vector and axial-vector couplings of the light quarks to the $Z$ boson, and $e_{q}$ is the charge of the quark of flavor $q$.

The charge-dependent polarization asymmetries of the NC cross sections are defined as

$$
A\left(e^{ \pm} p\right)=\frac{2}{P_{R}-P_{L}} \cdot \frac{\sigma^{ \pm}\left(P_{R}>0\right)-\sigma^{ \pm}\left(P_{L}<0\right)}{\sigma^{ \pm}\left(P_{R}>0\right)+\sigma^{ \pm}\left(P_{L}<0\right)} \simeq \mp k a_{e} \frac{F_{2}^{\gamma Z}}{F_{2}} .
$$

These asymmetries are proportional to the combinations $a_{e} v_{q}$ and thus provide a direct measure of parity violation. In the Standard Model $A\left(e^{+} p\right)$ is expected to be positive and about equal to $-A\left(e^{-} p\right)$. At large $x$ the asymmetries measure the $d / u$ ratio of the valence quark distributions according to

$$
A\left(e^{ \pm} p\right) \simeq \pm k \frac{1+d_{v} / u_{v}}{4+d_{v} / u_{v}}
$$



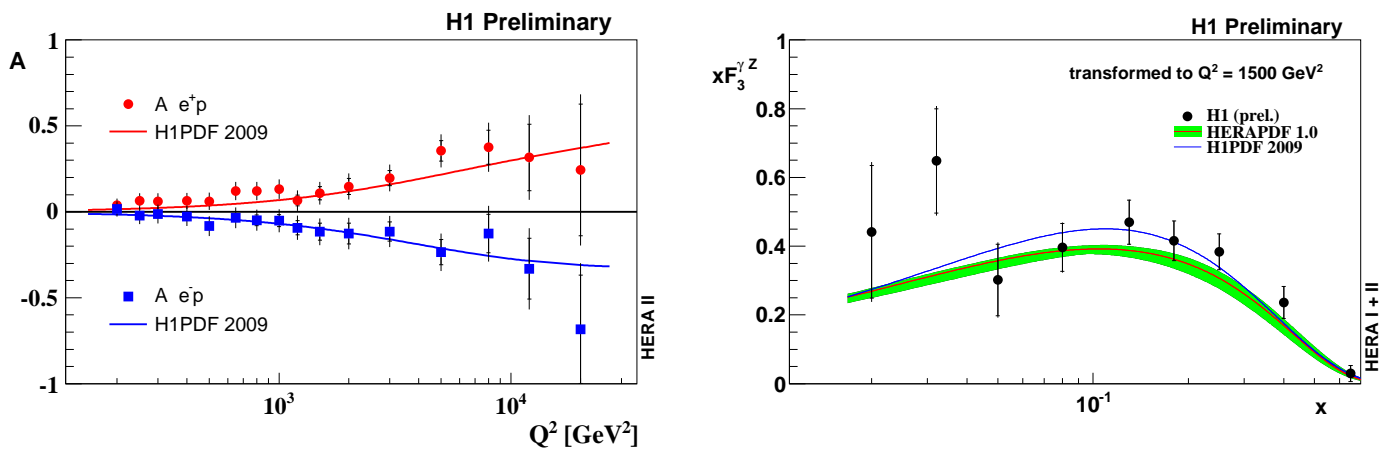

Figure 2: The H1 measurements of the polarization asymmetry in the $e^{+} p$ and $e^{-} p$ NC interactions (left) and of the structure function $x F_{3}^{\gamma Z}$ (right). The curves describe the SM predictions based on the NLO QCD fi ts $[3,5]$ to the unpolarized HERA-I data.

The results for the polarization asymmetries are shown in Fig. 2 (left). The polarization effects in the NC process are reliably established at large values of $Q^{2}$ corresponding to small distances. The asymmetries are well described by the SM predictions based on the NLO QCD fit [5] to the unpolarized HERA-I data.

A lepton beam charge dependence allows the structure function $x \tilde{F}_{3}$ to be measured using the combined unpolarized data from HERA-I and HERA-II. The structure function $x \tilde{F}_{3}$ can be obtained from the cross section difference between electron and positron data

$$
x \tilde{F}_{3}=\frac{Y_{+}}{2 Y_{-}}\left[\tilde{\sigma}_{N C}^{-}\left(x, Q^{2}\right)-\tilde{\sigma}_{N C}^{+}\left(x, Q^{2}\right)\right] .
$$

The dominant contribution to $x F_{3}$ arises from the $\gamma Z$ interference, which allows $x F_{3}^{\gamma Z}$ to be extracted according to $x F_{3}^{\gamma Z} \simeq-x \tilde{F}_{3} / k a_{e}$ by neglecting the pure $Z^{0}$ exchange contribution, which is suppressed by the small vector coupling $v_{e}$. The structure function $x F_{3}^{\gamma Z}$ is non-singlet and has little dependence on $Q^{2}$. The measured $x F_{3}^{\gamma Z}$ at different $Q^{2}$ values can thus be averaged taking into account the small $Q^{2}$ dependence. The averaged $x F_{3}^{\gamma Z}$, determined for a $Q^{2}$ value of $1500 \mathrm{GeV}^{2}$, is shown in Fig. 2 (right). The $x F_{3}^{\gamma Z}$ measurement, which is directly sensitive to the valence quark distributions, is well described by the NLO QCD fits $[3,5]$ to previously published HERA-I data.

\section{Electroweak and QCD fits at HERA}

The longitudinally polarized NC and CC cross section measurements from $\mathrm{H} 1$ and ZEUS are used in the combined electroweak and QCD fits to determine electroweak parameters. The parton distribution functions (PDFs) are evolved in $Q^{2}$ using the DGLAP equations in NLO from the distributions in $x$ defined at an initial scale $Q_{0}^{2}$. In the recent analysis of the $\mathrm{H} 1$ data $Q_{0}^{2}=1.9 \mathrm{GeV}^{2}$ and the PDF set $\left(x g, x u_{v}, x d_{v}, x \bar{U}\right.$ and $\left.x \bar{D}\right)$ are taken as in HERAPDF1.0 [3]. The H1 and ZEUS results [6] on the weak neutral couplings of $u$ and $d$ quarks to the $Z^{0}$ boson are shown in Fig. 3 in comparison with the LEP EW [7] and CDF [8] measurements. The polarization data improve the sensitivity to $v_{q}$ via $F_{2}^{\gamma Z}$ due to the term in Eq. 3.2 proportional to $P$. The HERA results are complementary and competitive with those determined from $e^{+} e^{-}$and $p \bar{p}$ experiments. 

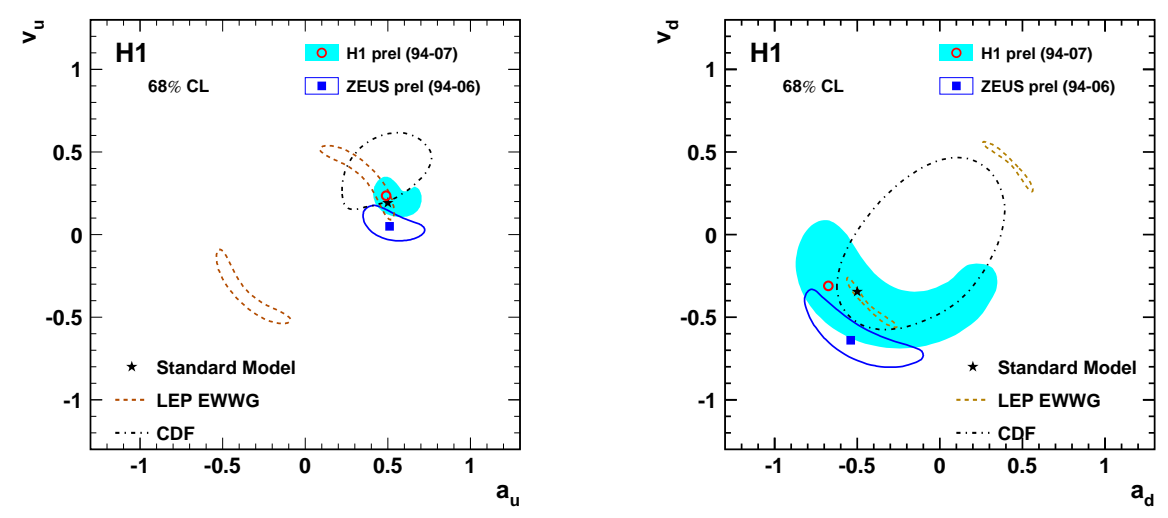

Figure 3: The $H 1$ and ZEUS results on the weak neutral couplings of $u$ and $d$ quarks to the $Z^{0}$ boson compared to measurements by the LEP EWWG and the CDF experiment.

\section{Summary}

The $e^{ \pm} p$ NC and CC cross sections are measured using the HERA-II data with longitudinally polarized lepton beams. The polarization dependence of the CC cross section exhibits the expected linear behavior, consistent with the absence of right-handed charged currents in the Standard Model. The polarization asymmetry of the NC cross section is obtained demonstrating parity violation at very small distances down to about $10^{-18} \mathrm{~m}$. The lepton beam charge dependence due to the interference between the photon and the $Z$ exchange is used to measure the interference structure function $x F_{3}^{\gamma Z}$ which is sensitive to the valence quark distributions. The polarized HERA-II data are used in the EW and QCD analyses, substantially improving the precision of the determination of the weak neutral couplings with respect to the previously published measurements based on the HERA-I data only. The results on $v_{q}$ and $a_{q}$ are complementary and competitive with those from $e^{-} e^{+}$and $p \bar{p}$ experiments.

\section{References}

[1] H1 Collaboration, H1prelim-09-042, H1prelim-09-043.

[2] S. Chekanov et al. et al. [ZEUS Collaboration], Phys. Lett. B 637, 210 (2006);

S. Chekanov et al. et al. [ZEUS Collaboration], Eur. Phys. J. C 61, 223 (2009), C 62, 625 (2009);

H. Abramowicz et al. [ZEUS Collaboration], DESY-10-129.

[3] F. D. Aaron et al. [H1 and ZEUS Collaborations], JHEP01, 109 (2010).

[4] W. F. L. Hollik, Fortschr. Phys. 38, 165 (1990).

[5] F. D. Aaron et al. [H1 Collaboration], Eur. Phys. J. C 64, 561 (2009).

[6] H1 Collaboration, H1prelim-10-042; ZEUS Collaboration, ZEUS-prel-07-027.

[7] LEP and SLD Electroweak Working Groups, Phys. Rept. 427, 257 (2006).

[8] D. E. Acosta et al. [CDF Collaboration], Phys. Rev. D 71, 052002 (2005). 